\title{
Reflections on Diagnosis and Diagnostic Errors: a Survey of Internal Medicine Resident and Attending Physicians
}

\author{
Thilan P. Wijesekera, MD, MHS๑, Lisa Sanders, MD, and Donna M. Windish, MD, MPH
}

Yale Department Internal Medicine, Yale University School of Medicine, New Haven, CT, USA.

J Gen Intern Med 35(2):614-5

DOI: $10.1007 / \mathrm{s} 11606-019-05045-\mathrm{z}$

(c) Society of General Internal Medicine 2019

\section{INTRODUCTION}

More than three years since the National Academy of Medicine (NAM) report "Improving Diagnosis in Health Care," providers and educators are still trying to understand why diagnostic errors occur and how to reduce them. ${ }^{1}$ In this survey study, internal medicine physicians share insights on how they make diagnoses and approach diagnostic error.

\section{METHODS}

From June 2016 through March 2017, we surveyed 484 residents and attendings from nine Connecticut internal medicine training programs. Surveys (electronic or paper) included single-answer, multiple choice questions about frequency of diagnostic uncertainty, diagnostic errors and considering harms and benefits of diagnostic testing (every patient, $\geq 2$ times per day, once daily, $\geq 2$ times per week, once weekly, once per month, and never), source of diagnostic errors (access, history, physical, testing, assessment, referral, follow-up), factors negatively impacting diagnosis (time, electronic health record and patient factors: literacy, language, mental health), and method of notification about diagnostic errors (family member, change in clinical status, laboratory testing, imaging, resident, attending, consultant, supervisor, institution/employer, ancillary staff). Analyses were performed using Pearson's $\chi^{2}$ test and summary statistics. Our IRB approved the study.

\section{RESULTS}

Surveys were completed by $55 \%(n=266)$ of physicians including $49 \%(n=196)$ of residents and $80 \%(n=70)$ of attendings. Respondents were primarily trainees $(n=196$,

$\overline{\text { Prior Presentations Oral Presentation: Diagnostic Error in Medicine 10th }}$ International Conference (10/8/2017).

Received April 10, 2019

Accepted April 24, 2019

Published online May 15, 2019
[74\%]), university-based ( $n=190,[71 \%])$, and of the female gender ( $n=87,[52 \%])$.

During the diagnostic process, time limitations ( $n=178$ [70\%]) most negatively impacted physicians' ability to make a diagnosis out of five key factors highlighted in the NAM report. Almost half of respondents in the inpatient $(n=127$ [49\%]) and outpatient $(n=95$ [41\%]) settings reported feeling diagnostic uncertainty at least daily (Fig. 1). Most inpatient $(n=174$ [67\%]) and outpatient $(n=143[61 \%])$ providers did not consider the harms and benefits of diagnostic testing with every patient.

Providers thought that diagnostic errors were uncommon, with the majority believing they occurred on a monthly basis or less $(n=136$ [54\%] inpatient; $n=134$ [60\%] outpatient) (Fig. 2). When they did occur, history $(n=98[38 \%])$ and assessment $(n=72[28 \%])$ were the most common sources of diagnostic error. Physicians reported most often being notified of a diagnostic error by a change in a patient's clinical status $(n=30$ [45\%] for attendings; $n=63$ [34\%] for residents).

Residents were less likely than attendings to always consider the harms and benefits of diagnostic testing in the inpatient $(63 \%$ vs. $77 \%, P=0.04)$ and outpatient (57\% vs. $76 \%, P=0.02$ ) settings. Female physicians thought diagnostic errors were less common than male physicians, reporting their occurrence on a monthly basis or less both inpatient ( $66 \%$ vs. $51 \%, P=0.05)$ and outpatient $(71 \%$ vs. $54 \%, P=0.04)$.

\section{DISCUSSION}

This needs assessment revealed key insights into the way internal medicine physicians approach the diagnostic process and diagnostic errors. Perhaps the most striking finding was that physicians often feel unsure of diagnoses, regardless of setting or experience. This concept of diagnostic uncertainty has gained increasing attention as providers navigate the challenges in medical decision making. ${ }^{2}$

One common challenge is time limitations, possibly due to high clinical demands, patient complexity, and administrative work. ${ }^{3,4}$ Despite these challenges, providers are not consistently weighing the harms and benefits of diagnostic testing, likely increasing the amount of overdiagnosis ${ }^{5}$ and 


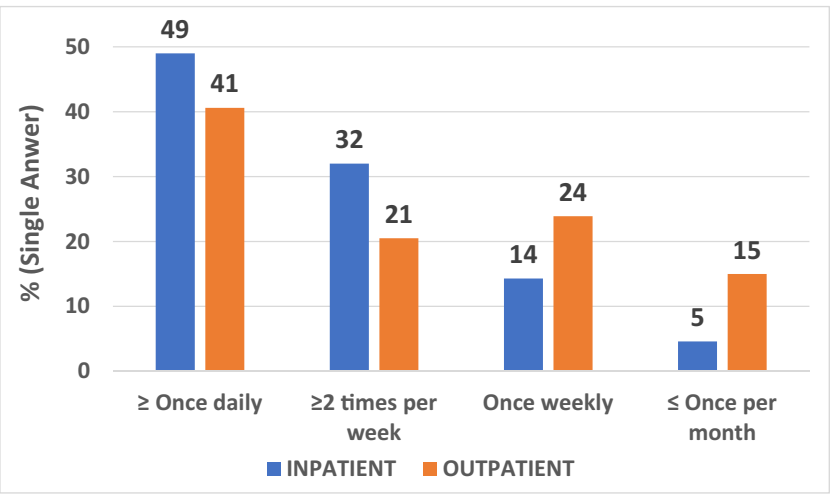

Figure 1 Self-reported frequency of diagnostic uncertainty among internal medicine physicians in the inpatient and outpatient settings.

necessitating initiatives like the Choosing Wisely Campaign $^{6}$ to facilitate cost-conscious care.

Given that diagnostic errors occur in $10-15 \%$ of patient encounters, ${ }^{1}$ physicians in our survey appear to underestimate the frequency of diagnostic errors with female physicians reporting fewer diagnostic errors, a controversial finding worth exploring in future studies. When diagnostic errors occur, they are usually attributed to history-taking or assessment and are most often recognized by a change in a patient's clinical status.

Study limitations include its survey format, which may not fully reflect provider behavior or quantify diagnostic errors.

In summary, internal medicine physicians in our study are often uncertain of a diagnosis yet underestimate the frequency of diagnostic errors and do not always consider the impact of diagnostic testing. Future quality improvement and medical education interventions should be directed at improving efficiency, increasing high-value care, and emphasizing clinical skills in patient care.

Corresponding Author: Thilan P. Wijesekera, MD, MHS; Yale Department Internal Medicine, Yale University School of Medicine, New Haven, CT, USA (e-mail: thilan.wijesekera@yale.edu).

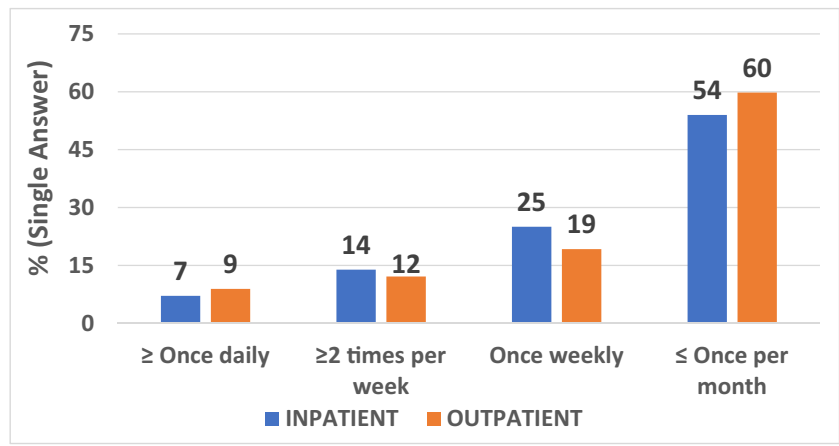

Figure 2 Self-reported frequency of diagnostic errors by internal medicine physicians in the inpatient and outpatient settings.

\section{Compliance with Ethical Standards:}

Conflict of Interest: The authors declare that they do not have a conflict of interest.

\section{REFERENCES}

1. National Academies of Sciences, Engineering, and Medicine. Improving diagnosis in health care. Washington, DC: National Academies Press: 2016.

2. Bhise V, Rajan SS, Sitting $\mathbf{D}$, et al. Defining and measuring diagnostic uncertainty in medicine: a systemic review. J Gen Intern Med. 2018;33(1):103-115.

3. Safford MM. The complexity of complex patients. J Gen Intern Med. 2015;30(12):1724-5.

4. Sinsky C, Colligan L, Li L, et al. Allocation of physician time in ambulatory practice: a time and motion study in 4 specialties. Ann Intern Med. 2016;165(11):753-60.

5. Brodersen J, Kramer BS, Macdonald H, Schwartz LM, Woloshin S. Focusing on overdiagnosis as a driver of too much medicine. BMJ. 2018;362:k3494.

6. Levinson W, Kallewaard M, Bhatia RS, et al. 'Choosing Wisely': a growing international campaign. BMJ Qual Saf. 2015;24(2):167-74.

Publisher's Note Springer Nature remains neutral with regard to jurisdictional claims in published maps and institutional affiliations. 